Available online at GSC Online Press Directory

GSC Biological and Pharmaceutical Sciences

e-ISSN: 2581-3250, CODEN (USA): GBPSC2

Journal homepage: https://www.gsconlinepress.com/journals/gscbps

(REVIEW ARTICLE)

\title{
Overview of ideal antilipidemic drugs: Past, present and the future
}

\author{
W Clement Atlee* \\ Assistant Professor, Department of pharmacology, C L Baid Metha College of pharmacy, Thoraipakkam, Chennai-97, \\ Tamilnadu, India.
}

Publication history: Received on 03 June 2020; revised on 11 June 2020; accepted on 15 June 2020

Article DOI: https://doi.org/10.30574/gscbps.2020.11.3.0162

\begin{abstract}
Atherosclerosis, a disease which affects large and medium sized arteries, is now a leading cause of death in many developed countries. High serum LDL (low density lipoproteins) and VLDL (very low density lipoproteins) levels are considered as atherogenic, whereas high HDL (High Density Lipoproteins) over $60 \mathrm{mg} / \mathrm{Dl}$ has a protective effect.Literature reviews were conducted by me from different articles on hypolipidemic drugs and the present review looking forward ideal hypolipidemic drugs with their target of action in the treatment of atherosclerosis.Statins are the first line antilipidemic drugs which possess dose dependent adverse effects like muscle pain, neuropathy, hepatic dysfunction and rhabdomyolysis. Reducing the statin dose, adding drug like proproteinconvertasesubtilisin/kexin type 9 inhibitors, supplements with coenzyme Q10 and L-carnitinie are some of the approaches to reduce statin advderse effects. In future for those patients who cannot tolerate statin and for those who can't achieve LDL target, squalene synthase inhibitors are the best choice. We focused on current existing hypolipidemic drugs, their targets and mechanism of actions and also the new ideal antilipidemic drugs of future. Ideal antilipidemic drug is one which should produce target lipid level, cause fewer side effects and drug interactions.
\end{abstract}

Keywords: Statins; Atherosclerosis; LDL; Novel drugs to treat hyperlipidemia

\section{Introduction}

Dyslipidemic persons have high risk for atherosclerosis (1). Atherosclerotic lesions form a localized plaque in intima and narrow the arterial lumen. High levels of triglycerides in plasma can cause pancreatitis. Accumulations of lipids in heart induce oxidative stress and inflammatory cardiac fibrosis leads to cardiac dysfunction [2].

Review starts with the different classes of lipoproteins with their role and normal and abnormal levels. Hyperlipidemia can be treated by life style changes, dietary modification, reducing the other risk factors of atherosclerosis and finally with effective and safe use of drug therapy.

Drug therapy start with statins monotherapy to reduce LDL-cholesterol. But inorder to reach LDL and triglycerides and HDL target in high risk cardiovascular patients combinations of drugs like ezetimibe, fibrates with statins play a important role. In patients who are intolerant to statins and those have constant elevation of triglycerides non-statin therapy with drugs like CETP inhibitors (Cholesteryl Ester Transfer Protein inhibitiors)) and PCSK9 inhibitors (Proprotein Convertase subtilisin/kexin type 9 inhibitors) are useful to reduce cardiovascular risk [3]. Effective and safe use of drugs and lower the lipoproteins level to the target level should be the motive in drug prescribing and drug usage by the patients. Starting with established frontline older antilipidemic drugs and development of newer novel ideal drugs in present and the future focused with the help of reference articles in this review.

\footnotetext{
*Corresponding author: W Clement Atlee Email: clementatlee2019@gmail.com
} 


\section{Plasma lipoproteins}

Water insoluble plasma lipids are transported in the following classes of lipoproteins.1. Chylomicrons 2. Chylomicron remnants 3. Very low density lipoproteins (VLDL) 4. Intermediate density lipoproteins (IDL) 5. Low density lipoproteins (LDL) 6. High density lipoproteins (HDL) 7. Lipoproteins (a) [4]

Table 1 Serum lipid levels (mg/dl) and the risk of ischemic heart disease

\begin{tabular}{lll}
\hline Lipid & Desirable level (Low risk) & Abnormal level (High risk) \\
\hline Total cholesterol & $<200$ & $>240$ \\
LDL cholesterol & $<130$ & $>160$ \\
HDL cholesterol & $>60$ & $<40$ \\
Triglycerides & $<200$ & $>400$ \\
\hline
\end{tabular}

\section{Hyperlipidemia}

An elevated level of lipids or lipoproteins in blood causes Hyperlipidemia.Hyperlipidemia is an important major risk factor for cardiovascular disease [5]. Reducing serum lipids can reverse this cardiac dysfunction.

\section{Management of Hyperlipidemia}

Stress reduction, dietary modification, reduce the risk factors of atherosclerosis like body weight, consumption of alcohol, smoking and treatment of diseases like hypothyroidism, diabetes mellitus, hypertension are also important while starting hypolipidemic drug therapy.

\section{Drug therapy}

\subsection{HMG CoA reductase (HydroxyMethylGlutaryl Coenzyme A reductase) inhibitors}

Statins by inhibiting HMG CoA reductase block HMG CoA to mevalonate conversion. This is the rate limiting step in cholesterol synthesis [6].

Reduced mortality rate and no tolerability issues are the main backbone reasons for statins's leading success rate over other non-statins in the treatment of atherosclerosis [7]. As per Scandinavian Simvastatin Survival Study statin reduced $35 \%$ LDL-cholesterol and 25\% Triglycerides after 5.4 years follow-up. But muscle related side effects are common in clinical practice while on statin therapy [8], [11]

Simvastatin possess antibacterial activity against staphylococcus aureus which has resistance to methicillin. Simvastatin because of its antibiofilm activity can be combined with topical antimicrobials to treat above methicilin resistant staphylococcus infection [9], [10]. Simvastatin can be considered as novel antibacterial agent.

Table 2 Currently used statins and their daily dose [12]

\begin{tabular}{cc}
\hline Statins & Daily dose in $\mathbf{~ m g}$ \\
\hline Lovastatin & $20-40$ \\
Pravastatin & $10-20$ \\
Simvastatin & $5-20$ \\
Fluvastatin & $20-60$ \\
Atorvastatin & $10-40$ \\
Rosuvastatin & $5-20$ \\
Pitavastatin & $1-4$ \\
\hline
\end{tabular}




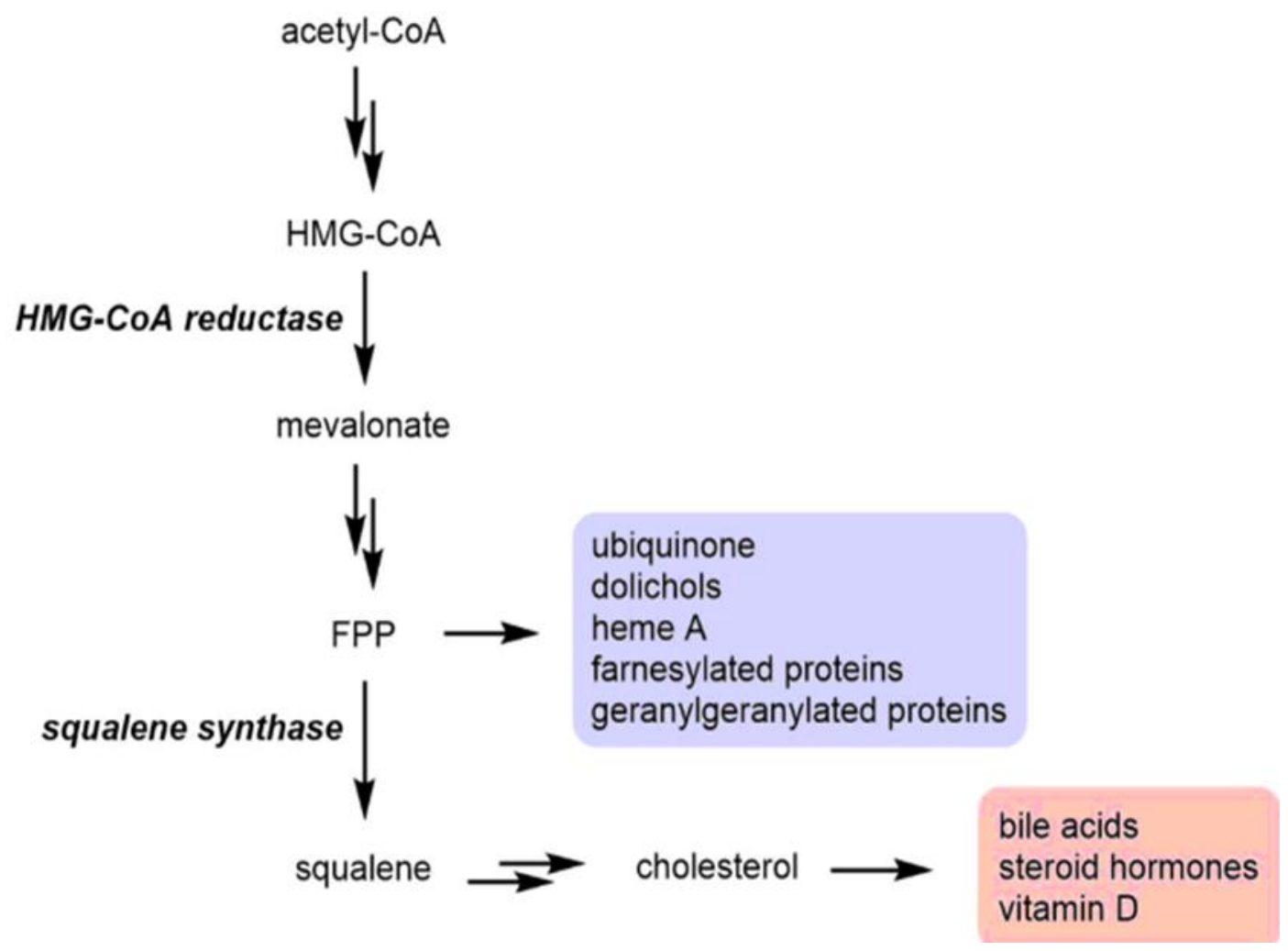

Figure 1Cholesterol Biosynthesis

[HMG-CoA: 3-hydroxy-3-methylglutaryl-CoA,

FPP: Farnesyl Pyrophosphate]

Adverse reactions of statins [12]

- Hepato toxicity-During statin therapy monitor transaminase level, if it reaches beyond more than three times of normal, discontinue statin therapy. Either shift to other drug or reduce the dose after transaminase level become normal.

- Muscle toxicity-Myopathy and muscular pains are caused by statins rarely. Elevated muscle enzymes level, rhabdomyolysis occur in few people. If patient get muscle pain it is better to check muscle enzyme levels.

- Diabetes- Before initiates statin therapy and during treatment with statin particularly with high dose checks blood sugar level.

- Cognitive impairment-Memory loss, mood changes and depression are some of the expected statin adverse reactions.

\subsection{Cholesterol absorption inhibitors}

Because of many patients have higher risk for coronary heart disease not reaching LDL goal after statin therapy alone, need a ideal second drug in addition with statins [13]. Also many patients meet intolerability issue when statins combined with fibrates, resins or niacin. Myopathy was severe when these drugs combined with statins. With combination of ezetimibe with statins no drug interactions are reported. Combination of ezetimibe and statin produces good cholesterol reduction than statins alone [16].

Ezetimibe decreases LDL cholesterol of plasma by 15-18\%, as monotherapy. Ezetimibe exhibit good safety profile. In the intestine ezetimibe inhibit both biliary as well as dietary cholesterol absorption.. Ezetimibe used in the dose range of 5-10 mg/day. Avoid its use in pregnancy and children [14], [15]. 


\subsection{PPAR $\alpha$ inhibitors}

Fibrates stimulate peroxisome proliferator activated receptor-alpha receptor which controls genes expression that mediate metabolism of triglycerides. Fibrates increase lipoprotein lipase and hydrolysis of triglycerides. Plasma triglycerides reduced by $50 \%$, cholesterol reduced by $15 \%$ and HDL increased by $20 \%$. Commonly used fibrates are gemfibrosil, bezafibrate and fenofibrate [17]. The novel drugs which target both alpha and gamma PPAR receptors improve both glycaemic and lipid parameters Example: Tesaglitazar [18].

Table 3 LDL cholesterol lowering efficacy of drugs [19]

\begin{tabular}{ll}
\hline Drug & Percentage LDL cholesterol reducing efficacy \\
\hline Statins & \\
Atorvastatin & $39-60$ \\
Rosuvastatin & $45-63$ \\
Bile acid sequestrants & $15-30$ \\
Niacin & $5-25$ \\
Fibrates & $5-20$ \\
Ezetimibe & 18 \\
\hline
\end{tabular}

Table 4 Triglycerides lowering efficacy of drugs [19]

\begin{tabular}{lc}
\hline \multicolumn{1}{c}{ Drug } & Percentage triglycerides lowering efficacy \\
\hline Fibrates & $30-50$ \\
Niacin immediate release & $20-50$ \\
Statins & $10-30$ \\
Ezetimibe & $5-10$ \\
Omega-3 fatty acids & $20-30$ \\
\hline
\end{tabular}

\subsection{Niacin}

Niacin in the dose of 2-8 g/day, decreases plasma triglycerides, LDL and raises HDL level. Niacin prevents lipolysis in adipose tissue. Slow down the fatty acids synthesis in liver. Side effect of niacin is flushing in therapeutic dose. But this can be minimized by taking niacin in smaller dose and increase gradually and administer with meals [20].

\subsection{Omega-3 Fatty acids}

Omega-3 fatty acids in fish oil decrease triglycerides and have no drug interaction with statins. Recommended dose is 2-4 gram/daily. Patients with very high triglycerides level above or equal to $500 \mathrm{mg} / \mathrm{DL}$ have more risk for acute pancreatitis [21].

\subsection{ACAT inhibitor (Acyl- Coenzyme A: Cholesterol acyl Transferase inhibitor)}

ACAT is the enzyme which synthesise cholesterol esters in tissues. ACAT inhibitor avasimibe reduce plasma cholesterol level and suppress atherosclerosis. Rall FJ et al enrolled twenty seven subjects in a double blind randomized 3-sequence cross over trial and administered atorvastatin $80 \mathrm{mg}$ QD, avasimibe $750 \mathrm{mg}$ QD and combined treatment of atorvastatin $80 \mathrm{mg}$ QD and avasimibe $750 \mathrm{mg}$ QD after washout period of four weeks. Six week treatment period alloted for each treatment [22]. After combination therapy Total cholesterol level was reduced by $22 \%$ instead of $18 \%$ by atorvastatin monotherapy. 


\subsection{Bile acid transport inhibitor}

Metabolic effect of Elobixibat, a bile acid transporter inhibitor was evaluated by Rudling, M et al. [23]. After four week study on dyslipidemic patients drug effect on plasma lipids and bile acid synthesis was evaluated in samples. LDL cholesterol was reduced by $7.4 \%$ and bile acid synthesis was also induced.

Because elobixibat inhibit apical sodium dependent bile acid transporter, ileal bile acid absorption is reduced. Colon motility is increased due to more colon content of bileacid. Elobixibat reduces chronic constipation and the cardiovascular risk. Elobixibat should be taken before breakfast and tolerated upto 20 mg dose [24].

\subsection{CETP inhibitor ( Cholesteryl Ester Transfer Protein inhibitor )}

CETP inhibitors reduce serum low-density lipoproteins and elevate high density lipoprotein cholesterol [25] and reduced the incidence of diabetes (12\%). Cholesterol esters are transferred from HDL by CETP to LDL, forms lipoprotein apo-B which leads to more peripheral arterial deposition of cholesterol in walls. CETP inhibitors by increasing the HDL concentration reduced the incidence of diabetes. After anacetrapib treatment there is a modest cardiovascular benefit compared to the other two CETP inhibitors.

Table 5 CETP inhibitors and their properties [26]

\begin{tabular}{llll}
\hline Drug & Dose & LDL-C reduction & Increase in HDL \\
\hline Torcetrapib & $60 \mathrm{mg} /$ day & $25 \%$ & $72 \%$ \\
Dalcetrapib & $600 \mathrm{mg} /$ day & No change & $31 \%$ \\
Anacetrapib & $100 \mathrm{mg} /$ day & $40 \%$ & $138 \%$ \\
Evacetrapib & $130 \mathrm{mg} /$ day & $36 \%$ & $129 \%$ \\
\hline
\end{tabular}

\subsection{Lipoprotein(a) inhibitor}

Elevation of lipoprotein(a), an atherogenic lipoprotein act as a genetic risk marker for atherosclerosis[27]. Manocha A and Srivastava LM explained benefit of lowering of lipoprotein (a) in treatment of atherosclerosis. Inhibition of Lipoprotein (a) by inhibiting lipoprotein (a) assembly is a better choice in therapy of future.

Niacin in high dose and PCSK9 inhibitors have the ability to lower lipoprotein (a) but not yet proved by trials.The ability of antisense oligonucleotides to lower lipoprotein (a) level near to normal level is a great scope in future to reduce cardiovascular risk in patients after the ongoing clinical trials [28].

\subsection{Novel drugs with other mode of treatments to treat dyslipidemia}

Other than the first line statins therapy the new treatments with novel drugs with other mode of actions are needed in order to improve efficiency to reach target LDL-cholesterol in high risk patients, or as alternative in statin intolerants or add on therapy with statins[29].

\subsubsection{PCSK9 inhibitors [30]}

PCSK9 (Proprotein Convertase subtilisin/kexin type 9) inhibitors

PCSK9 promote LDL receptor destruction on liver cells, prevent blood clearance of LDL.

Table 6 PCSK inhibitors and their brand names and dose [31]

\begin{tabular}{lll}
\hline Drug & Brand name & Dose \\
\hline Alirocumab & Praluent (sanofil) & 75 mg every two weeks, Subcutaneously \\
Evolocumab & Repatha (amgen) & 140 mg every two weeks \\
\hline
\end{tabular}

Whenever we double the dose of statins to lower LDL, only $6 \%$ additional lowering effct of LDL we are getting. But with high statin dose we get more increase in transaminase level and muscle toxicity as adverse effect. Achieving target LDL 
level in cardiovascular risk patients have become increasingly complex. Addition of PSSK9 inhibitors with low dose stains avoiding the stain side effects and also reduces LDL effectively [32].

\subsubsection{MTP (Microsomal Triglyceride transfer Protein) Inhibitor [33]}

MTPI are able to reduce both triglycerides and LDL-cholesterol. Lomitapide from Aegerion, a MPTI able to reduce LDLcholesterol. Lomitapide monotherapy at $5 \mathrm{mg}$ for four weeks reduced $19 \% \mathrm{LDL}$ and $30 \%$ at $10 \mathrm{mg}$. $60 \mathrm{mg}$ is the maximum dose. Hepatic steatosis and increase in liver transaminase level are the serious side effects. Plasma concentration of statin and warfarin can be increased when they are used along with lomitapide.

Maximum Assembly of apolipoproteins, triglycerides and cholesterol in liver is the role of MTP [34].

\subsubsection{AMPK/ATP-citrate lyase (AMP-activated protein kinase and ATP-citrate lyase)}

ETC-1002 (8-hydroxy-2, 2, 14, 14-tetramethylpentadecanedioic acid), a novel investigational drug inhibit ATP citrate lyase. ATP citrate lyases an enzyme coordinate in lipid synthesis. Combination of these two mechanisms regulates LDL [35].

ETC-1002 (bempedoic acid) and ezetimibe combination is a better option to reduce LDL in statin intolerant patients. Increased side effects with increase in statin dose, many patients not able to reach LDL target are the reasons for the clinical trials focusing ezetimibe and ETC-1002 combination or statin and ETC-1002 combination [36].

\subsubsection{Apolipoprotein B synthesis inhibitor $[37,40]$}

Apolipoprotein B is important protein component of lipoproteins like VLDL and LDL. Mipomerson reduce atherogenic lipids and lipoproteins in patients with hypercholesterolemia by inhibit apolipoprotein B. Once in a week $200 \mathrm{mg} / \mathrm{ml}$ subcutaneously is the recommended dose. Elevation in plasma alanine aminotransferase level and fat accumulation in liver are the side effects. In severe liver or renal dysfunction avoid mipomerson.

\subsubsection{Squalene synthase inhibitor}

HMG CoA reductase inhibitors act by reducing mevalonate production. But non-sterols like coenzyme Q10 production depend on mevalonate. This causes elevation of hepatic transaminase level. Because squalene synthase inhibitors inhibit cholesterol synthesis (Figure-1) beyond mevalonate production, squalene synthase inhibitors become the best novel antilipidemic drug. Unlike statins hepatic transaminase level elevation is only moderate with squalene synthase inhibitors [38, 39 and 41].

\section{Conclusion}

Statin use is contraindicated in pregnant women and in acute liver disease. Ezetimibe monotherapy can be started but in active liver disorder avoid ezetimibe with statin combination. Bile acid sequestrants like colestipol should be avoided in biliary or bowel obstruction. Avoid fibrates in severe liver or renal disorders. For childrens below 10 years ezetimibe usage is not advisable. Among statins use Pitavastatin can be considered because of its less drug interaction. Colesevelam is the only lipid lowering drugs approved by FDA to use safely during pregnancy and also in children. PCSK9 inhibitors cannot be used in hypersensitivity patients. Cost of novel drugs PCSK9 inhibitors has their limitations in use. Novel drugs like CETP inhibitors and PPAR gamma inhibitors have apart from antilipidemic action also have antidiabetic potential. Novel Squalene synthase inhibitors produce decreased rise in hepatic transaminase level as adverse effect as compare to statins.We need more novel drugs which reduce blood LDL and triglycerides, increase HDL and to reach lipid goal in risky patients. But research should go on not only targeting disease but also their safe usage in other comorbid conditions, in pregnancy, childrens and old people.

\section{Compliance with ethical standards}

\section{Acknowledgments}

I wish to thank C.L. Baid Metha College of Pharmacy management and principal madam for motivating me to write this review.

\section{Disclosure of conflict of interest}

This review article is completely devoid of conflict of interest. 


\section{References}

[1] Kennedy. C, Koller Y, Surkova E. (2020). Effect of coenzyme Q10 on statin-associated myalgia and adherence to statin therapy: A systematic review and meta-analysis. Atherosclerosis. 299, 1-8.

[2] Yao, Y.S., Li, T.D. \& Zeng, Z.H. (2020). Mechanisms underlying direct actions of hyperlipidemia on myocardium: an updated review. Lipids in Health and Disease, 19(23).

[3] Sando KR and Knight M. (2015). Non-statin therapies for management of dyslipidemia: a review. Clinical Therapeutics, 37(10), 2153-2179.

[4] RS Satoskar, Nirmala NR, Raakhi KT and SD Bhandarkar.(2017). Pharmacology and pharmacotherapeutics, 25th Ed. popular prakashan publication, 590-8.

[5] Nelson RH. (2013). Hyperlipidemia as a risk factor for cardiovascular disease. Primary Care, 40(1), 195-211.

[6] Jiang, S., Li, H., Tang, J. et al. (2018). Discovery of a potent HMG-CoA reductase degrader that eliminates statininduced reductase accumulation and lowers cholesterol. Nature Communications, 9, 5138.

[7] Toth PP, Banach M. (2019). Statins: Then and now. Methodist Debakey Cardiovascular Journal, 15(1), 23-31.

[8] Golomb BA, Evans MA. (2008). Statin adverse effects: A review of the literature and evidence for a smitochondrial mechanism. American Journal of Cardiovascular Drugs, 8(6), 373-418.

[9] Thangamani S, Mohammad H, Abushakba MF, Hamed MI, Sobreira TJ, Hedrick VE, Paul LN, Seleem MN. (2015). Exploring simvastatin, an antihyperlipidemic drug, as a potential topical antibacterial agent. Scientific Reports, $5,16407$.

[10] Connie BN, David P, Jonathan AT, Terry AJ, Robert L, Larry BG et al. (2019). Statin safety and associated adverse events: A scientific statement from the American Heart Association. Arteriosclerosis Thrombosis and Vascular Biology, 39, e38-e81.

[11] Spence JD and Dresser GK. (2016). Overcoming challenges with statin therapy. Journal of the American Heart Association, 5(1).

[12] Rhee EJ, Kim HC, Kim JH, Lee EY, Kim BJ and Kim EM et al. (2019). 2018 Guidelines for the management of dyslipidemia in korea. The Korean Journal of Internal Medicine, 34(5), 1171.

[13] N Stone. (2002). Combination therapy: its rationale and the role of ezetimibe. European Heart Journal supplements, 4, J19-J22.

[14] Al-Shaer, M.H., Choueiri, N.E. \& Suleiman, E.S. (2004). The pivotal role of cholesterol absorption inhibitors in the management of dyslipidemia. Lipids in Health and Disease, 3, 22.

[15] E. Leitersdorf. (2001). Cholesterol absorption inhibition: filling an unmet need in lipid-lowering management. European Heart Journal Supplements. 3 suppl_E, E17-E23.

[16] Brown WV. (2003). Cholesterol absorption inhibitors: defining new options in lipid management. Clinical Cardiology, 26, 259-264.

[17] Toma A. (2013). Recent advances on novel dual-acting peroxisome proliferator-activated receptor alpha and gamma agonists. International Journal of Pharmaceutical Sciences and Research, 4(5), 1644-1653.

[18] Fagerberg B, Edwards S, Halmos T, et al. (2005). Tesaglitazar, novel dual peroxisome proliferator-activated receptor alpha/gamma agonists, dose-dependently improves the metabolic abnormalities associated with insulin resistance in a non-diabetic population, Diabetologia, 48(9), 1716-1725.

[19] Barter PJ, Rye KA. (2016). New era of lipid-lowering drugs, Pharmacological Reviews, 68(2), 458-475.

[20] Nelson RH. (2013). Hyperlipidemia as a risk factor for cardiovascular disease, Prime Care, 40(1), 195-211.

[21] Ann CS, Peter WFW, William SH, Eliot AB, Penny MK and Chesney KR et al. (2019). Omega-3 fatty acids for the management of hypertriglyceridemia: A science advisory from the American Heart Association. Circulation, 140(12), e673-e691.

[22] Raal FJ, Marais AD, Klepack E, Lovalvo J, Mclain R, Heinonen T. (2003). Avasimibe, an ACAT inhibitor, enhances the lipid lowering effect of atorvastatin in subjects with homozygous familial hypercholesterolemia. Atherosclerosis, 171(2), 273-279. 
[23] Rudling, M., Camilleri, M., Graffner, H. et al. (2015). Specific inhibitions of bile acid transport alter plasma lipids and GLP-1. BMC Cardiovascular Disorders, 15(75).

[24] Kumagai Y, Amano H, Sasaki Y, Nakagawa C, Maeda M, Oikawa I, Furuie H. (2018). Effect of single and multiple doses of elobixibat, an ileal bile acid transport inhibitor, on chronic constipation: A randomized controlled trial. British Journal of Clinical Pharmacology, 84(10), 2393-2404.

[25] Walter Masson, Lobo Martin Lobo, Daniel Siniawski, Melina Huerin, Graciela Molinero, et al. (2018). Therapy with cholesteryl ester transfer protein (CETP) inhibitors and diabetes risk. Diabetes and Metabolism, 44(6), 508-513.

[26] Mohammadpour AH and Akhlaghi F. (2013). Future of cholesterol ester transfer (CETP) inhibitors: a pharmacological perspective. Clinical Pharmacokinetics, 52(8), 615-626.

[27] Manocha A and Srivastava LM. (2016). Lipoprotein (a): a unique independent risk factor for coronary artery disease. Indian Journal of Clinical Biochemistry, 31(1), 13-20.

[28] Tsimikas S. (2016). Lp(a) as a new target for reduction of risk of cardiovascular disease and emergence of novel therapies to lower Lp(a). Current Opinion in Endocrinology Diabetes and Obesity, 23(2), 157-164.

[29] Kramer W. (2016). Antilipidemic drug therapy today and in the future. Handbook of Experimental Pharmacology, 233, 373-435.

[30] Hegele RA and Tsimikas S. (2019). Lipid- lowering agents. 124(3), 386-404.

[31] Zodda D, Giammona R and Schifilliti S. (2018). Treatment strategy for dyslipidemia in cardiovascular disease prevention: Focus on old and new drugs. Pharmacy (Basel), 6(1), 10.

[32] Chaudhary R, Garg J, Shah N and Sumner A. (2017). PCSK9 inhibitors: A new era of lipid lowering therapy. World Journal of Cardiology, 9(2), 76-91.

[33] Claudia Stefanutti. (2014). Targeting MTP for the treatment of homozygous familial hypercholesterolemia. Journal of Clinical Lipidology, 9(3), 369-381.

[34] Davis KA and Miyares MA. (2014). Lomitapide: A novel agent for the treatment of homozygous familial hypercholesterolemia. American Journal of Health-System Pharmacy, 71(12), 1001-1008.

[35] Pinkosky. SL, Filippov S, Srivastava RA, et al. (2013). AMP-activated protein kinase and ATP-citrate lyase are two distinct molecular targets for ETC-1002, a novel small molecule regulator of lipid and carbohydrate metabolism. Journal of Lipid Research, 54(1), 134-151.

[36] Bilen 0 and Ballantyne CM. (2016). Bempedoic acid (ETC-1002): an investigational inhibitor of ATP citrate lyase. Current Atherosclerosis Reports, 18(10), 61.

[37] Bell DA, Hooper AJ and Burnett JR. (2011). Mipomersen, an antisense apolipoprotein B synthesis inhibitor. Expert Opinion on Investigational Drug, 20(2), 265-272.

[38] Charlton-Menys V, Durrington PN. (2007). Squalene synthase inhibitors: clinical pharmacology and cholesterollowering potential. Drugs, 67(1), 11-16

[39] Reshma GB, Nadar MSAM, Selvakumar PM. (2018). A review on anticholesterol drugs and their mechanisms. Journal of Medicinal Chemistry and Drug Design, 1(1).

[40] Shattat G.F. (2014). A review article on hyperlipidemia: Types, treatments and new drug targets.Biomedical and Pharmacology Journal, 7(2).

[41] Abd-Alkareem HF, Jaber AH, Ibrahim AQ and Fanok MA. (2020). Lipid lessening drugs overview. GSC Biological and Pharmaceutical Sciences, 10(01), 90-95.

\section{How to cite this article}

Atlee WC. (2020). Overview of ideal antilipidemic drugs: Past, present and the future. GSC Biological and Pharmaceutical Sciences, 11(3), 67-74. 